\title{
La Identidad Digital del alumnado universitario: estudio descriptivo en la Facultad de Educación de la Universidad de Murcia
}

\section{Digital identity of university students: A descriptive study in the Faculty of Education of the University of Murcia}

\author{
Francisco Luis Saorín Sánchez \\ Universidad de Murcia \\ franciscoluis.saorin@um.es \\ Isabel Gutiérrez Porlán \\ Universidad de Murcia \\ isabelgp@um.es
}

Recibido: 20/07/2017

Aceptado: 23/02/2018

Publicado: 29/06/2018

\section{RESUMEN}

Este artículo aborda el fenómeno de la Identidad Digital y cómo éste es percibido por los estudiantes universitarios. La investigación realizada ha sido un estudio descriptivo contextualizado dentro de la metodología cuantitativa, con una muestra participante compuesta por 69 estudiantes pertenecientes todos ellos a la Facultad de Educación de la Universidad de Murcia.

Para conseguir nuestros objetivos, desarrollamos nuestro propio cuestionario atendiendo a los trabajos de Castañeda y Camacho (2012), Pérez (2012) y Gamero (2009). El cuestionario fue validado mediante un Juicio de expertos y se aplicó de forma telemática. Entre los resultados más destacados encontramos que los estudiantes tienden a interactuar en Internet menos de lo esperado. También hemos encontrado que tener bloqueados contactos es una actitud ligada al desarrollo de su interacción social y que los universitarios no necesitan conocer a un contacto en la vida real para establecer una conexión emocional con ellos.

\section{PALABRAS CLAVE}

Identidad digital; estudiantes universitarios; redes sociales.

\section{ABSTRACT}

This article focuses on the phenomenon of the Digital Identity and how it is perceived by university students. The research made is a descriptive study contextualized inside a quantitative methodology. 69 students from the Faculty of Education in the University of Murcia have participated.

For the achievement of our research goals, we developed our own questionnaire, bearing in mind the investigation of Castañeda y Camacho (2012), Pérez (2012) and Gamero (2009). The questionnaire was validated and delivered though the Internet. Among the results, we found out that the sample tends to interact in the Internet less than we expected. Having blocked contacts is a really common attitude in the developing of their social interaction and, finally, University Students doesn't need to meet a contact in face to face environments to connect emotionally with them. 


\section{KEYWORDS}

Digital identity; University students; social networks.

\section{CITA RECOMENDADA}

Saorín, F. L. Gutiérrez, I. (2018). La identidad digital del alumnado universitario: estudio descriptivo en la Facultad de Educación de la Universidad de Murcia. RIITE. Revista Interuniversitaria de Investigación en Tecnología Educativa, 4, 82-93. Doi: http://dx.doi.org/10.6018/riite/2018/30001

\section{Principales aportaciones del artículo y futuras líneas de investigación:}

- Visión transversal de lo que los universitarios de la Facultad de Educación de la Universidad de Murcia piensan sobre su Identidad Digital.

- Propuesta de gestión de la Identidad Digital.

- Investigación cuantitativa sobre las verdaderas tendencias de los estudiantes con respecto a su propia Identidad Digital con una muestra mayor.

- Investigación cuantitativa sobre el concepto de Identidad Digital que poseen los docentes desde una triple perspectiva: su identidad como individuos, docentes e investigadores.

\section{INTRODUCCIÓN}

La llegada de las nuevas Tecnologías de la Información y la Comunicación (conocidas por sus siglas TIC) ha ejercido tal influencia, que ha modificado todos los ámbitos de la sociedad con los que ha entrado en contacto y su irrupción ha traído consigo el cambio en la forma que teníamos de comunicarnos. Toda la actividad que desarrollamos en la Red ofrece información que nos define como individuos, esto es, muestra nuestra Identidad Digital (ID de ahora en adelante).

Para Castañeda y Camacho (2012), la ID no es tanto la idea de una nueva identidad, sino la propia dentro de un mundo digitalizado. Esto quiere decir que solamente es el reflejo de nosotros mismos dentro de un mundo virtual. La ID que se nos plantea tiene 3 dimensiones claramente definidas: lo que yo dejo ver de mí, a quién reflejo y en quién me reflejo. La primera, la parte más personal que constituye nuestra ID, es la única que está en nuestra mano. En cuanto a los otros dos ámbitos, los cuales no podemos manejar directamente, también aportan información a nuestra identidad. El primero se refiere a los amigos que nos rodean y el segundo a nuestros seguidores. La ID es una construcción que realizamos mediante hechos que queremos destacar de nuestra identidad offline o de lo que otros usuarios opinan de nosotros con la idea de que nos represente dentro del mundo virtual (García, 2010). Por otro lado, Wood y Smith (2005) amplían la definición del concepto al tomarlo como una estructura compleja, basada en quien creemos ser, en cómo queremos que los demás nos perciban y en cómo realmente nos perciben.

Para gestionar la ID Gamero (2009) presenta algunas herramientas con las que el individuo puede empezar a crear y gestionar su ID dentro del mundo virtual: Blogs, Microblogs, Sitios Webs, Redes Sociales, Correo electrónico y Material Multimedia. Si, por el contrario, no queremos sobreexponer nuestros datos en la Red, existen diferentes webs que nos permitirán descubrir cuánto de nosotros hay disponible. Estas webs son Pipl, 123people y Yasni. También debemos destacar la herramienta llamada $\mathrm{Me}$ on the web, la cual nos alertará mediante notificaciones a nuestro correo si nuestro nombre, apellido o dirección de correo queda expuesto en la Red (Oficina de Seguridad del Internauta, 2011). 
El sociólogo Dubar (2002) afirma que las estructuras tradicionales sobre la identidad están en decadencia, ya que éstas no sirven en la actualidad para definir el mundo actual, a los demás, y ni siquiera a nosotros mismos. Almansa, Fonseca y Castillo (2013) aseguran que, para los jóvenes, la identidad es un tema muy importante y por eso dedican bastante tiempo a diseñarla, compartiendo en las Redes información del tipo: selfies, fechas de nacimiento (algunas falsas), lugar de residencia, dónde han estudiado e, incluso, cuáles son su música o programas de tv favoritos. Cáceres, Ruiz y Brändle (2009) arrojan un poco de luz sobre el comportamiento de los jóvenes en las Redes Sociales con relación a la mentira. El uso de identidades falsas está denostado socialmente, por lo que suelen afirmar con rotundidad que amigos y conocidos suyos lo practican, pero a la hora de hablar de ellos mismos, suelen ser más esquivos, dando normalmente una respuesta evasiva siempre negativa. Sin embargo, para Schouten (2007) los jóvenes usan la Web para experimentar con su identidad, encontrándose un alto \% de situaciones en donde han pretendido mostrarse como algo que no eran.

Tal y como afirma White (2010) hay dos tipos de individuos con respecto al uso de las TIC: los Visitantes y los Residentes. Los primeros son aquellos que usan las Redes de forma ocasional y siempre para hacer una tarea en concreto, mientras que los segundos (donde se encuentran los jóvenes nativos digitales) pasan la mayor parte del día en Internet y sienten especial preocupación por su ID.

La ID ha sido tema de estudio en múltiples investigaciones, arrojando los resultados obtenidos un poco de luz tanto a la propia definición del concepto como al efecto que ésta tiene sobre determinadas muestras seleccionadas. Según García (2010) dichas investigaciones comenzaron hace más de 20 años, primero en MUDs y Chat Rooms (donde se ocultaba la identidad o se pretendía pasar por otra persona), luego en la arcaica Web 1.0 y, finalmente, en la Web 2.0.

Por todo ello hemos considerado de vital importancia realizar una investigación al respecto, la cual consiga arrojar un poco de luz sobre la visión que poseen los universitarios de la Facultad de Educación de la Universidad de Murcia acerca de su propia ID. Para, seguidamente, ofrecerles un decálogo que les ayude a gestionarla de forma óptima.

\section{MÉTODO}

Las preguntas de las que parte nuestra investigación son las siguientes:

¿Son conocedores los estudiantes universitarios de la importancia que tiene la Identidad Digital?

¿Siguen los alumnos universitarios algún proceso para gestionar su Identidad Digital?

\subsection{Objetivos}

El objetivo general que pretendemos alcanzar con esta investigación es el de "Conocer la Identidad Digital de los estudiantes universitarios y la importancia y valor que éstos otorgan a la misma". Aunque para ello, este objetivo se concreta en los siguientes objetivos específicos:

- Objetivo específico 1: Explorar y analizar el concepto de Identidad Digital, sus principales características y los espacios en Red en los que puede desarrollarse la ID.

- Objetivo específico 2: Describir la ID de los estudiantes universitarios de la Facultad de Educación de la Universidad de Murcia.

- Objetivo específico 3: Diseñar una propuesta de gestión de la ID orientada a los estudiantes universitarios. 


\subsection{Diseño de la investigación}

El problema que hemos decidido investigar ha sido la percepción que tienen los universitarios sobre su propia ID. Según Monje (2011) hay múltiples maneras de investigar la realidad social, aunque dentro de las Ciencias Sociales, las más comunes son: el paradigma cuantitativo y el cualitativo; y cada una de ellas tiene características, metodologías e instrumentos propios. En nuestro caso nos hemos decidido por la corriente cuantitativa y, dentro de ella, por la realización de un estudio descriptivo. Para obtener los datos hemos diseñado una encuesta como instrumento de recogida de información, la cual una vez respondida, sería tratada mediante el programa Google Formularios.

\subsection{Definición de la muestra y su contexto}

Para la realización de este proyecto hemos contado con una muestra $(n=69)$, pertenecientes todos ellos a alguna de las carreras ofertadas por la Facultad de Educación de la Universidad de Murcia. El muestreo utilizado para la investigación ha sido no probabilístico, más concretamente, muestreo por conveniencia, el cual consiste en elegir una muestra de la población por su accesibilidad. Este tipo de muestreo viene perfecto para aquellas situaciones en las que no existen diferencias entre los individuos cercanos y el conjunto de la población. Como contrapunto negativo, señalamos que impide poder realizar generalidades con rigor estadístico sobre la población (Ochoa, 2015).

\subsection{Diseño del instrumento de recogida de información}

Para la realización de esta investigación sobre la ID en los universitarios nos hemos servido de la técnica de la encuesta para poder obtener información al respecto sobre la muestra, los alumnos de la Facultad de Educación de la Universidad de Murcia.

Tras revisar la bibliografía pertinente acerca de la ID y no encontrar cuestionarios que se adaptaran al tipo de información que se quería analizar en la investigación, hemos tenido que desarrollar nuestro propio instrumento. Para ello hemos tenido en cuenta a todos los autores consultados, pero, especialmente, a Castañeda y Camacho (2012), Pérez (2012) y Gamero (2009). Tras llevar a cabo el diseño del instrumento, éste fue sometió a una validación realizada por 4 expertos en Tecnología Educativa.

Dicha validación se llevó a cabo vía email, haciéndoles llegar un documento con la encuesta y otro con una rúbrica. En la hoja de evaluación las preguntas se organizaron en campos temáticos y fueron evaluadas atendiendo a su comprensión-univocidad, su pertinencia y su importancia. En esa misma tabla, cada pregunta tenía un apartado llamado Observaciones, en el que los expertos podían aportar sugerencias o explicar el porqué de su valoración. Con todas las respuestas que fueron llegando, se fue modificando el instrumento de recogida de información para hacerlo lo más efectivo y riguroso posible.

El cuestionario ha sido configurado en base a 30 preguntas en las que se abordan diferentes temáticas relacionadas con la ID. Así, podemos encontrar preguntas centradas tanto en el número de perfiles y cuentas que poseen en la Red como el tiempo que le dedican y el uso que hacen de ellos. Las preguntas, diseñadas siempre teniendo en cuenta su concisión y claridad, fueron organizadas en dos tipos: de selección múltiple (que solo admitían una única respuesta) y casillas de verificación (las cuales aceptaban más de una respuesta simultáneamente).

\subsection{Tratamiento de datos}

Para el estudio análisis de los datos, en nuestra investigación hemos hecho uso de la aplicación Google Formularios. Todas las variables con las que vamos a trabajar tienen una naturaleza cualitativa nominal, esto quiere decir que "presenta modalidades no numéricas que no admiten un criterio de orden" (Vitutor, 2014). Dentro de este tipo de variables nos 
encontraremos con que son variables categóricas, las cuales irán graficadas mediante sectores circulares y de barras.

\section{RESULTADOS}

Con respecto a los datos sociodemográficos, el cuestionario fue respondido por una muestra de 69 individuos, de los cuales, el $75,4 \%$ representaban al sexo femenino y el $24,6 \%$ al sexo masculino. Todos los participantes, en el momento de realizarse la investigación, se encuentran matriculados en una de las diversas carreras ofertadas por la Facultad de Educación de la Universidad de Murcia de la siguiente forma: Un 46,4\% son estudiantes de Educación Primaria, el 13\% corresponde a los estudiantes de Educación Social, el 27,5\% son estudiantes de Educación Infantil y el $13 \%$ son alumnos de Pedagogía. Finalmente, y ahora poniendo nuestro foco de atención en el año académico que se encuentran cursando en el momento de la encuesta, comprobamos que están matriculados en distintos cursos: un $37,7 \%$ son de $1^{\circ}$ Curso, un $31,9 \%$ es alumnado de $2^{\circ}$, del $3^{\circ}$ año han respondido un $13 \%$ y de $4^{\circ}$ Curso un $17,4 \%$.

Con respecto a la pregunta sobre en cuáles espacios en Red tienen abierto un perfil, la opción más votada ha sido "redes sociales" con un 98,6\%. Muy próxima a ésta nos encontramos la opción "correos electrónicos" con $97,1 \%$. Las opciones "blogs", "página web" y "medios sociales" han recibido $53,6 \%, 11,6 \%$ y $58 \%$ respectivamente. Sin embargo, la opción "no utilizo ningún espacio en Red" no ha recibido ninguna puntuación. Para concretar más, se quiso saber cuáles eran esas plataformas en Red en las que ellos habían abierto un perfil. De esta forma pudimos saber que la opción "whatsapp" ha sido la opción más votada, obteniendo un $100 \%$ de votos, lo que se traduce en que los 69 encuestados la puntuaron. Muy cerca de ésta, podemos encontrar "Facebook" con un $84,1 \%$, "youtube" con 79,7\% e "Instagram" con un $78,3 \%$.

Figura 1. Medios Sociales empleados.

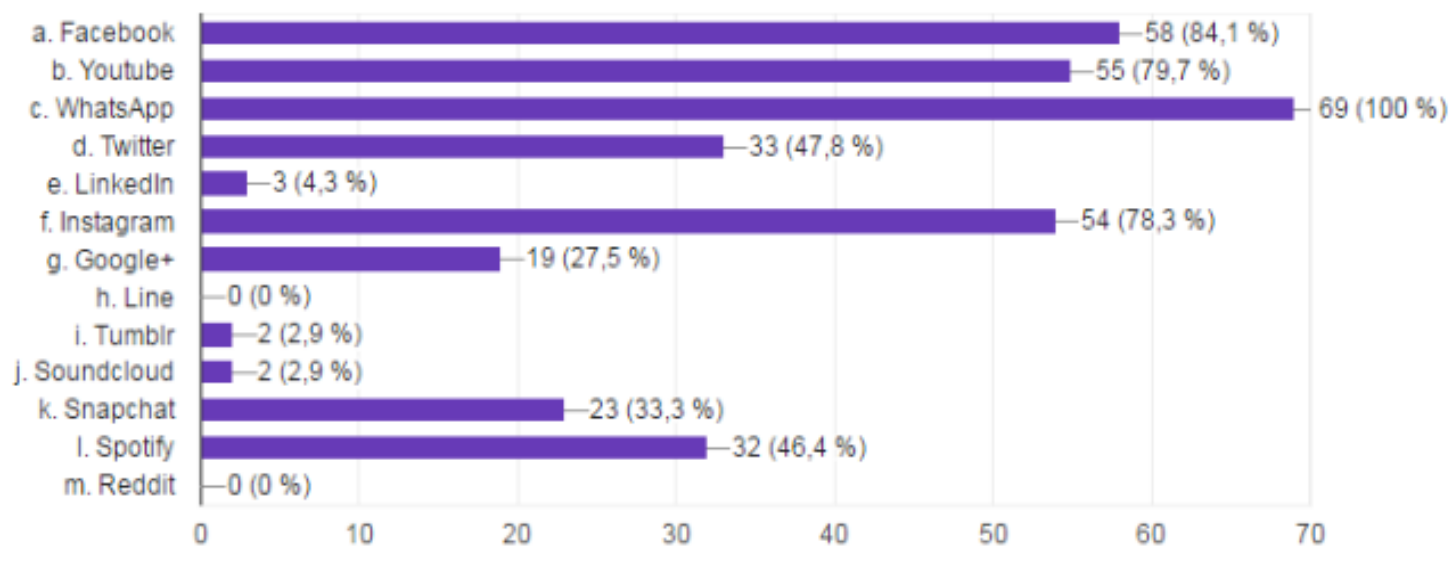

Una vez que ya sabemos el tipo de espacio en red que prefieren, así como las Redes Sociales en las que prefieren estar presentes, el siguiente paso será conocer la frecuencia con la que actualizan sus perfiles. Un $23,2 \%$ de los encuestados afirman hacerlo "algunas veces", mientras que un $23,2 \%$ afirma hacerlo "bastante". Finalmente, un $4,3 \%$ se posicionan en el valor de la escala más alto "mucho" mientras que un $27,5 \%$ han optado por la opción "B. Poco". Curiosamente, ninguno de los encuestados ha seleccionado la opción "A. Nada".

La siguiente cuestión se centra sobre la forma en la que tienden a identificarse en la Red. Aquí vemos que el $53,6 \%$ se presenta "con su verdadero nombre", mientras que un $4,3 \%$ afirma que "con un Nick". Por lo que, como vemos, existe un $42 \%$ de los encuestados que dice 
hacer uso indistintamente de uno o de otro, añadiendo que "depende del tipo de Red" para usar una opción u otra.

Sea haciendo uso de un usuario (nick) o de su verdadero nombre, lo que sí sabemos es que el tiempo que dedican al uso de las Redes Sociales es de un $42 \%$ para la opción "entre 2-4 horas", un $26,1 \%$, "entre $4-6$ horas", un $14,5 \%$ "más de 7 horas" y un 17,4\% para "no más de 2 horas al día". En ese tiempo que gastan en la Web, podemos ver que la información que suelen publicar en sus perfiles, tal y como indica la mayoría, son: "fotos" (88,4\%), "estados" $(52,2 \%)$, "enlaces relevantes" $(47,8 \%)$ y "reflexiones personales" $(36,2 \%)$.

Figura 2. Tipo de información que suelen publicar.

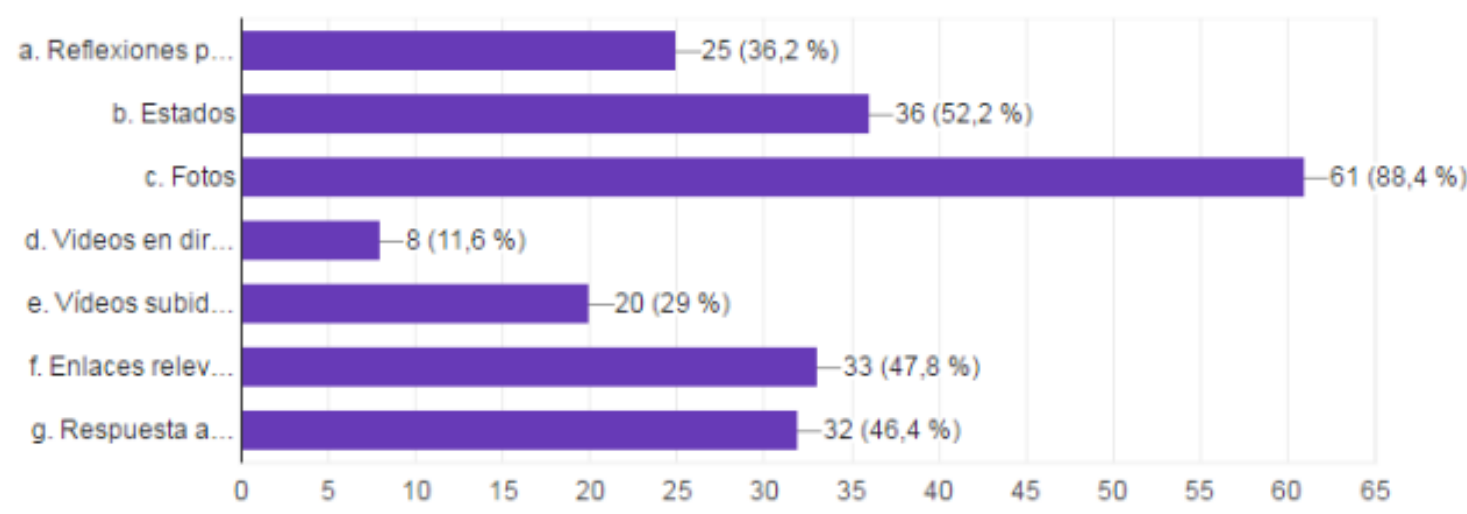

De acuerdo con la siguiente respuesta, el número de post que suelen compartir en un día suele ser de "entre 0 y 1 " con un $72,5 \%$, "Entre 2 y 4 " con un $21,7 \%$ y, finalmente, "más de 4 " con un $5,8 \%$. Si esas publicaciones que han realizado, son opiniones, la encuesta nos muestra que la opción "política" ha obtenido un $18,8 \%$, "fútbol" un $21,7 \%$, "reflexiones sobre la vida" $55,1 \%$, "moda" un $23,2 \%$, "religión" un 7,2\% "humor" un 60,9\%, "trabajo" un $21,7 \%$, "escuela" $53,6 \%$ y "otros" un $44,9 \%$.

Con respecto al tipo de datos que suelen compartir en sus Redes Sociales, podemos ver que la opción "fecha/lugar de nacimiento" ha obtenido un $84,1 \%$, "número de teléfono" un $11,6 \%$, "nombre y apellidos completos" un $59,4 \%$, "formación académica" un $68,1 \%$, "fotos y vídeos privados" un $44,9 \%$ y "lo que estoy haciendo" un $33,3 \%$.

Los encuestados, dentro del ámbito social que poseen las Redes, creen que las publicaciones que más reacciones generarían entre sus contactos serían "Un Selfie" con un $43.5 \%$ de votos, "Un estado/post" con un $36,2 \%$, "Un video" con un $46,4 \%$, y finalmente, para el $43,5 \%$ "Un meme". Con respecto a la frecuencia con la que suelen recibir comentarios un 5,8\% afirma que "Nada", un $29 \%$ habla de "Poco", el 50,7\% señala que "algunas veces"; mientras que para "Bastante" y "Mucho" tenemos un $14,5 \%$ y un $0 \%$ respectivamente. En el caso contrario, a la pregunta sobre la frecuencia con la que los encuestados comentan en publicaciones de otros usuarios, el 43,5\% afirma que lo hace "Algunas veces" mientras que el $36,2 \%$ dice que "Poco". Así mismo, Un 13\% ha votado por la opción "Bastante" y un 5,8\% y un $1,4 \%$ para las opciones "Nada" y "Mucho" respectivamente.

Centrándonos ahora en la relación que establecen los encuestados con sus contactos vemos que a la pregunta sobre si alguna vez les han hecho comentarios en Redes Sociales que no les han gustado hemos obtenido un $72,5 \%$ que ha marcado la opción "No" y un "27,5\%" que ha marcado la opción "Sí". Recíprocamente, a la pregunta sobre si han herido alguna vez a alguien con algún comentario hemos obtenido un $89,9 \%$ que ha marcado la opción "No" y un $10,1 \%$ que ha marcado la opción "Si". 
Atendiendo a las respuestas obtenidas a la pregunta sobre si se han arrepentido alguna vez de haber subido alguna foto/comentario a la Red hemos obtenido un 55,1\% que ha marcado la opción "Sí" y un "44,9\%" que ha marcado la opción "No". Estas respuestas se encuentran estrechamente ligada a la pregunta sobre si creen que la información que comparten puede afectar a tu futuro laboral. En esta cuestión, hemos obtenido un 53,2\% que ha marcado la opción "No", un 18,8\% que ha marcado la opción "Hasta hora no lo había pensado" y un "29\%" que ha marcado la opción "Si". Preguntándoles a continuación sobre si han buscado empleo mediante las Redes hemos obtenido un $69,6 \%$ que ha marcado la opción "No" y un "30,4\%" que ha marcado la opción "Sí". Con respecto a si lo han encontrado mediante dichas herramientas, comprobamos que un $89,9 \%$ ha marcado la opción "No" y un "10,1\%" ha marcado la opción "Sí".

De acuerdo al número de contactos que los encuestados poseen en la Red Social que más utilizan, los resultados obtenidos han sido los siguientes: Un 18,8\% dice tener "Menos de 100 contactos", "Entre 100-300" hay un 37,7\%, un 27,5\% afirma tener "Entre 300-600" y "Más de 600 " un $15,9 \%$.

De todos esos contactos que poseen en sus Redes, preguntamos únicamente por aquellos que tienen en la aplicación Facebook, obteniendo que un 30,4\% de los encuestados afirma conocer a todos sus contactos en la vida real, el $42 \%$ afirma que "Sí, aunque tengo unos pocos contactos que no conozco personalmente", y el 18,8\% "Sí, aunque tengo bastantes contactos que no conozco personalmente". Debemos señalar que la opción "No conozco a ninguno de mis contactos" obtuvo una puntuación del $0 \%$.

Figura 3. Relación con sus contactos.

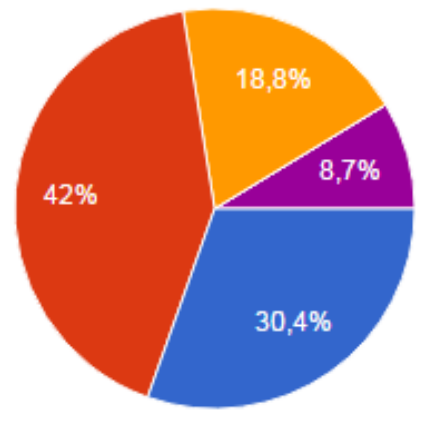

Sí, a todos

Sí, aunque tengo unos pocos contactos que no conozco personalmente

Sí, aunque tengo bastantes contactos que no conozco personalmente

No conozco a ninguno de mis contactos en la vida real

No tengo cuenta en Facebook

Dentro de esos contactos que afirman conocer, nos interesa saber si les une a ellos algún lazo sanguíneo, por lo que a la pregunta sobre si poseen contactos de familiares en sus redes un $69,6 \%$ ha respondido que "toda la familia al completo", un 15,9\% han dicho que "sólo hermanos", un $11,6 \%$ señaló "núcleo familiar" y, finalmente, un 2,9\% dicen no tener a ningún miembro. A continuación, realizamos la misma pregunta, pero ahora nos interesa saber si también tienen como contactos a compañeros de trabajo y de la universidad. Las respuestas obtenidas han sido un $97,1 \%$ que ha marcado la opción "sí" y un "2,9\%" que ha marcado la opción "no". Finalmente, y con respecto a la relación entre la muestra y los contactos "incómodos", un $33,3 \%$ dice no tener a ninguno bloqueado, mientras que el $66,7 \%$ afirma lo contrario.

Con respecto a la valoración que los encuestados dan a las opiniones de otros usuarios, sobre todo cuando van a adquirir un producto o un servicio, el $65,2 \%$ se decanta por la opción "Bastante", un 15,9\% para la opción "mucho" al igual que la opción "poco". Finalmente, la 
opción menos votada ha sido "nada", con un 2,9\%. Al contrario, si preguntamos la frecuencia con la que ellos dejan reseñas sobre la adquisición de algún artículo a fin de poder ayudar a otros usuarios, un $53,6 \%$ ha respondido "alguna vez", un $34,4 \%$ dicen que "nunca, y finalmente, un $4,3 \%$ y un $7,2 \%$ señalan "siempre" y "casi siempre", respectivamente.

A la pregunta sobre si han mentido alguna vez por internet sobre ellos y su identidad, un $42 \%$ ha respondido "alguna vez" y un $58 \%$ ha señalado "nunca". Curiosamente, las opciones "siempre" y "casi siempre" no han sido seleccionadas. Por último, a la cuestión acerca del uso que dan a sus Redes Sociales hemos obtenido que un $84,1 \%$ las usan para "estar en contacto con amigos", un 10,1\% las usan "para hacer nuevos amigos" y un 5,8\% para "promoción académica".

\section{DISCUSIÓN Y CONCLUSIONES}

Según Aleixandre y Ferrer (2010) el hombre siempre ha tenido la predisposición natural de comunicarse y compartir sus experiencias con los demás, por lo que las nuevas tecnologías, y sobre todo las que tienen un origen en la Web 2.0, han ayudado a cubrir esa necesidad. Muchas veces, la sensación de seguridad y tranquilidad que nos brinda la red, hace que entreguemos a nuestro buscador demasiada información sensible sobre nosotros (Garay, 2016). Esa información que inconscientemente ofrecemos contiene una parte muy importante de nosotros, pues nos identifica como individuos dentro de la Red. Dicha información, dependiendo de cuál sea su origen, pueden ser: formales, informales, reales o imaginarios (Omatos y cuevas, 2011).

Con respecto al primer objetivo específico que delimitamos al principio, sabemos que en palabras de Castañeda y Camacho (2012) Pérez (2012) y Gamero (2009) la Identidad Digital es la suma de la información que obtenemos de perfiles, comentarios, mails y contenidos digitales (Blogs, sitios webs, RRSS, correos electrónicos y material multimedia). La identidad no es ajena a nosotros, sino que es nuestro propio yo dentro de un entorno virtual y se resume en: lo que dejo ver de mí, a quién reflejo y en quién me reflejo.

Aunque la muestra analizada $(n=69)$ no permita establecer generalidades en los resultados obtenidos, sí es cierto que su estudio puede arrojar un poco de luz a la cuestión que centra nuestro objetivo №2. De acuerdo con lo que dejamos ver de nosotros cuando accedemos a la Red, podemos comprobar que para los alumnos universitarios las Redes Sociales y el Correo Electrónico es la herramienta que más se ajusta a sus demandas y necesidades de interacción para con sus iguales. Curiosamente, y por obvio que pueda parecer, ninguno de los encuestados ha afirmado no poseer algún perfil. Esta situación refuerza lo planteado por Área (2011), quien afirmaba que no estar presente en los medios digitales sería como renunciar a una parte importante de nuestra propia identidad. Del tiempo dedicado al manejo y gestión de sus redes, afirman estar entre 2 y 4 horas conectados, pero solo actualizan su perfil algunas veces y suelen subir entre 0 y 1 contenido al día, por lo que se deduce que el resto de horas lo dedican a visitar otros perfiles y páginas de interés. De esta visita a perfiles de otros usuarios, podemos ver que los encuestados no muestran especial interés en dejar comentarios en entradas, ya que la mayoría afirma comentar en pocas ocasiones.

García (2010) sostiene que los jóvenes hacen un uso de su ID de manera favorecedora, de tal forma que nunca va en detrimento de ellos, mostrándose una realidad parcial en donde se destacan los aspectos positivos. Nuestra investigación demuestra que dicha reflexión también se cumple, ya que los encuestados prefieren compartir mayormente contenido de humor o reflexiones personales que tratar temas de política, trabajo o religión, mucho más susceptibles éstos a crear desavenencias en la red. Con respecto a las mentiras empleadas en la web, la mayoría ha negado haber mentido en Internet sobre su identidad. 
Como sabemos, el uso de identidades falsas está denostado socialmente, por lo que suelen afirmar con rotundidad que amigos y conocidos suyos lo practican, pero a la hora de hablar de ellos mismos, suelen ser más esquivos, dando siempre una respuesta evasiva siempre negativa (Cáceres, Ruiz y Brändle, 2009). Lo mismo ocurre con el uso del nombre de usuario. Muy pocos han dicho emplearlos en todas sus redes. La mayoría ha afirmado identificarse en las Redes con su verdadero nombre y, los que no, han optado por la respuesta evasiva que sostenía el uso, según el tipo de red utilizada.

Atendiendo al número de contactos, vemos que no abundan los perfiles con un número muy elevado, cosa que facilita bastante su gestión. Analizando el tipo de contactos que tienen los encuestados resulta fácil comprender el carácter social que adquieren los espacios en Red, ya que la gran mayoría tiene entre sus "amigos" a compañeros de clase y del trabajo, así como a miembros de su familia. García (2010) afirma que la presencia de los universitarios en las Redes Sociales busca la sociabilidad. En este sentido, podríamos añadir y completar que los jóvenes buscan la sociabilidad y evitan el conflicto; siendo éste el principal motivo por el que la gran mayoría de la muestra encuestada afirma tener contactos bloqueados.

Con respecto a la muestra seleccionada, vemos también que no tienen especial interés en el acto de comentar. Sobre la pregunta acerca de si suelen dejar comentarios en publicaciones de otros usuarios las respuestas mayoritarias han demostrado que no suelen hacerlo con mucha frecuencia. Esta misma situación aparece con respecto a la pregunta sobre el número de comentarios que suelen recibir, las cuales también reflejan una baja actividad. Este planteamiento choca un poco con la idea generalizada que se suele tener de los jóvenes, ya que la bibliografía al respecto los presenta como individuos que viven en un entorno tecnológico en donde llevan a cabo sus relaciones sociales y aquí vemos que no, que ese aspecto nuestra muestra no lo cubre y se desentiende bastante. De los pocos mensajes que dicen recibir, un número significativo, aunque no mayoritario, reconoce haber recibido comentarios hirientes y despectivos y de los pocos que afirman escribir, un porcentaje todavía más pequeño sostiene haber herido a alguien mediante un comentario en la Red.

Eso referente a los comentarios en perfiles personales en redes, pero ¿Qué ocurre con los comentarios y reseñas sobre productos? La muestra los valora positivamente, esto es, los tienen en cuenta para tomar sus decisiones. Curiosamente, no ocurre lo mismo a la inversa. A la pregunta sobre si son ellos los que dejan comentarios vemos que los resultados indican que no lo hacen o lo hacen muy poco. Aquí volvemos a ver un poco de dejadez en su comportamiento, ya que sabiendo lo útiles que son, no están interesados en participar y ayudar a otros usuarios con sus reseñas y opiniones. Atendiendo a la esfera laboral, vemos que, aunque se venden las maravillas de la Web 2.0 para encontrar trabajo muy pocos son los que las han utilizado, pero lo que todavía llama más la atención es que si reducido es el número de usuarios de ese tipo de aplicaciones menor es el número de los que afirman haber encontrado empleo. Aunque si observamos su respuesta acerca del principal uso que dan a sus Redes Sociales, el buscar empleo no está en los planes de ningún participante.

Como ya hemos mencionado, el hecho de haber realizado un estudio descriptivo, de haber realizado un muestreo por conveniencia y de manejar una muestra relativamente pequeña no nos permita establecer grandes generalidades, podemos decir que el estudio ofrece una visión de los universitarios un poco alejada de lo que se suele pensar. Aunque en ciertos comportamientos si encajan con lo esperado, como el caso de la temática que tratan sus posts (entradas) o de su innegable presencia en la Red mediante el uso de perfiles, resulta sorprendente la falta de interacción entre ellos, por lo que se desglosaba de los datos de la investigación. Aunque una abrumadora mayoría afirma que usan las Redes para estar en contacto con sus amigos, luego vemos que la interacción entre ellos es bastante escasa.

Con la intención de dar respuesta al objetivo específico número 3 vemos que autores como Valerio, Castro y Herrera Murillo (2016) señalan que, aunque los estudiantes 
universitarios valoran poseer una buena ID, los resultados de las investigaciones muestran que apenas reflexionan sobre las actividades que llevan a cabo en internet. Dicho planteamiento ha quedado totalmente refutado por nuestra investigación ya que, tal y como hemos podido comprobar en el apartado "amenazas" de nuestro análisis DAFO, vemos que los encuestados hacen un uso de las redes sin realizar ninguna reflexión previa sobre su actuación.

Acerca de la pregunta sobre si creen que la información que comparten en la Red les puede perjudicar en su entrada al mundo laboral, si juntas las opciones de "sí" y la de "hasta ahora no lo había pensado" vemos que se queda sensiblemente cerca de la respuesta mayoritaria, "no". Esto sugiere que existe un conflicto, que hay jóvenes que están seguros de lo que suben y comparten y creen que no les compromete en nada y hay otros que o no han reflexionado lo suficiente o que directamente lo creen. Esto refuerza lo apuntado por Castañeda y Camacho (2012) quienes señalan la idea de que los universitarios conocen, o llegan a imaginar, el efecto que supone la información depositada en internet acerca de ellos; pero no les importa demasiado.

Como "Debilidad" obtuvimos la cierta dejadez mostrada por parte de los encuestados acerca de la configuración de su perfil, así como el poco interés que tienen en compartir contenidos, por lo que, deducimos que la difusión de información no se encuentra entre los principales motivos por los que han decidido tener presencia en la red (de ahí la falta de reflexión sobre lo que suben a Internet). Nuestra encuesta arroja algo de luz sobre esta cuestión, afirmándose que la principal finalidad que buscan en la red es la de mantener una relación con sus contactos. Aunque, también debemos señalar que, a pesar de mostrar desapego y falta de reflexión, gran parte de los encuestados afirma haberse arrepentido alguna vez de su actuación dentro del mundo virtual.

Con respecto a la forma que tienen de interactuar, nuestro análisis DAFO destaca como aspecto positivo la educación que dicen poseer cuando se relacionan en Internet, y es que, según los datos de la encuesta, no suelen mentir asiduamente, ni hacen comentarios hirientes a otros usuarios. Esta característica, resulta muy beneficiosa a la hora de construir una Identidad, ya que tener una correcta reputación, fuera de cualquier polémica, ofrece una sensación de confianza en los demás. Así mismo, y continuando todavía dentro del apartado "Fortalezas" de la investigación, hemos obtenido información relevante acerca de la tendencia hacia la homogeneización en el uso de determinados espacios en red que presentan los encuestados. $Y$, en parte tiene una notoria razón de peso: como hemos comprobado, los universitarios presentan como finalidad su presencia en la red a establecer lazos sociales; por lo que un elemento facilitador de éstos es la cohabitación en un mismo entorno. Por este motivo, la mayoría de los encuestados delimita su presencia a Redes Sociales, y dentro de ellas, a Facebook, Whatsapp e Instagram.

Dentro del apartado de las "Oportunidades" debemos destacar como algo favorable, el reducido número de contactos, los cuales, para la mayoría, no sobrepasan los 300 amigos. Es muy importante saber quiénes son los que ven la información que compartimos en la web, por lo que un número pequeño de seguidores nos permite gestionar mejor lo que cada uno puede ver de nosotros sin perder la perspectiva. El hecho de que la mayoría de contactos sean de personas conocidas en la vida real, como hemos comprobado con el análisis, ayuda a que no exista ese desfase entre la identidad analógica y la digital, ya que esos contactos están presentes y conocen ambas.

Una vez que hemos realizado nuestro análisis DAFO y que hemos comprobado qué es lo que opinan determinados autores sobre lo que hay que tener en cuenta a la hora de gestionar nuestra ID, estamos preparados para diseñar un decálogo que facilite la gestión de una identidad dentro de la Red: 
1- Elige cuidadosamente el espacio en Red en el que quieres estar presente.

2- Mantén una buena reputación y ayuda a otros usuarios a fortalecer la suya, compartiendo siempre información veraz y contractada referente a ellos.

3- Utiliza programas para comprobar qué es lo que los demás pueden ver de ti en la Red.

4- Mantén actualizada tu presencia en la Red.

5- Tener contactos conocidos de nuestra vida real en nuestras Redes Sociales.

6- Utiliza varias cuentas o perfiles, para evitar que la esfera personal y la profesional entren en contacto.

7- Reflexionar siempre antes de colgar un post o compartir una imagen.

8- Evita siempre exponer fotos e información privada o comprometida.

9- Evita tener perfiles públicos, o al menos, no compartir información sensible en ellos.

10- Evita entrar en disputas, descalificaciones y peleas con otros usuarios.

Con toda la información obtenida de nuestra investigación podemos afirmar que, a la pregunta sobre si son conscientes de la importancia de conlleva tener una determinada ID en la web, los alumnos de la Facultad de Educación de la Universidad de Murcia tienen una actitud desfavorable al respecto. Aunque los resultados han demostrado que hacen un uso responsable, vemos que no lo hacen con la intención de preservar su intimidad; sino que no lo hacen por simple desidia. Así mismo, y al respecto de si siguen algún proceso para gestionarla, vemos que apenas hay una actitud crítica frente a sus actividades desarrolladas dentro de la web.

\section{ENLACES}

Acceso al instrumento de recogida de información utilizado en este estudio: https://is.gd/ntsQ3e

\section{REFERENCIAS BIBLIOGRÁFICAS}

Aleixandre, R., y Ferrer, A. (2010) ¿Qué nos aportan las Redes Sociales? Anuario ThinkEPI, 4, 217-223. Recuperado de https://dialnet.unirioja.es/descarga/articulo/3263918.pdf

Almansa, A., Fonseca, O., y Castillo, A (2013). Redes sociales y jóvenes. Uso de Facebook en la juventud colombiana y española. Comunicar: revista científica iberoamericana de comunicación y educación, 1 (40),127-135. doi: http://dx.doi.org/10.3916/C40-2013-03-03

Area, M. (2011). TIC, identidad digital y educación. Cuatro reflexiones. Reencuentro, (62), 9799. Recuperado de http://www.redalyc.org/articulo.oa?id=34021066012

Cáceres, M. D., Ruiz J. A., y Brändle, G. (2009). Comunicación interpersonal y vida cotidiana. La presentación de la identidad de los jóvenes en Internet. CIC. Cuadernos de Información y Comunicación, 14, 213-231. Recuperado de http://revistas.ucm.es/index.php/CIYC/article/view/CIYC0909110213A/7226

Castañeda, L., y Camacho, M. (2012) Desvelando nuestra identidad digital. El profesional de la información, 21(4), 354-360. doi: https://doi.org/10.3145/epi.2012.jul.04

Dubar, C. (2002). La crisis de las identidades. La interpretación de una mutación. Barcelona: Edicions Bellaterra.

Gamero, R. (2009). La configuración de la identidad digital. Enter-IE 131. Recuperado de: https://observatorio.iti.upv.es/media/managed files/2009/06/03/11569.pdf

Garay, V. (2016). ¿Para qué necesitamos anonimato y por qué es importante defenderlo?. Recuperado de https://www.derechosdigitales.org/10211/para-que-necesitamos-anonimatoy-por-que-es-importante-defenderlo/ 
García, K. (2010). Construcción de la identidad digital de los estudiantes javerianos en Facebook. Trabajo de grado para optar por el título de Comunicadora social Campo Profesional Editorial. Universidad javeriana, Bogotá.

Monje, C.A., (2011). Metodología de la investigación cuantitativa y cualitativa. Guía didáctica. Neiva: Colombia: Universidad surcolombiana.

Ochoa, C. (2015). Muestreo no probabilístico: muestreo por conveniencia. Recuperado de https://www.netquest.com/blog/es/blog/es/muestreo-por-conveniencia

Oficina de Seguridad informática (2011). Tu identidad digital. Recuperado de https://www.osi.es/es/actualidad/blog/2011/06/21/tu-identidad-digital

Omatos, A., y Cuevas V. (2011). Identidad Digital y Redes Sociales con menores. Recuperado de https://sites.google.com/site/tallerid11/identidad-digital

Pérez, M. (2012). Introducción. Identidad digital. TELOS, (91), 55-59.

Schouten, A. (2007). Adolescents' online self-disclosure and selfpresentation. Amsterdam, Print Partners Ipskamp, Enschede.

Valerio, G., Castro, H., y Herrera Murillo, D. (2016). El rol de la identidad digital de los universitarios en el ingreso a la vida laboral. Revista Espacios, 37(16), 6. Recuperado de http://www.revistaespacios.com/a16v37n16/16371606.html

Vitutor (2014). Variable estadística. Recuperado de http://www.vitutor.com/estadistica/descriptiva/a 2.html

White, D. (2010). Visitors and Residents. Recuperado de https://www.youtube.com/watch?time continue $=17 \& v=0$ sFBadv04eY

Wood, A., y Smith, M. (2005). Online communication: linking technology, identity, and culture. Mahwah, New Jersey: Lawrence Earlbaum.

\section{INFORMACIÓN SOBRE LOS AUTORES}

\section{Francisco Luis Saorín Sánchez \\ Universidad de Murcia}

Graduado en Educación Primaria por la Universidad de Murcia con Mención en Lengua Extranjera Inglés. Pronto amplió sus conocimientos realizando un Máster interuniversitario en Tecnología Educativa: eLearning y Gestión del Conocimiento. Actualmente cuenta en el mercado con varias obras didácticas, creadas para facilitar el aprendizaje del Inglés y la Música, sirviéndose de las nuevas tecnologías como soporte y difusión.

\section{Isabel Gutiérrez Porlán}

Universidad de Murcia

Profesora Contratada Doctora del Departamento de Didáctica y Organización Escolar en la Facultad de Educación de la Universidad de Murcia. Miembro del Grupo de Investigación de Tecnología Educativa de la Universidad de Murcia. Licenciada en Pedagogía por la Universidad de Murcia. Master en Tecnología Educativa: aprendizaje virtual y gestión del conocimiento por la Universidad de las Islas Baleares. Doctora en Tecnología Educativa por la Universidad Rovira i Virgili.

\footnotetext{
Los textos publicados en esta revista están sujetos a una licencia de Reconocimiento 4.0 España de Creative Commons. Puede copiarlos, distribuirlos, comunicarlos públicamente y hacer obras derivadas siempre que reconozca los créditos de las obras (autoría, nombre de la revista, institución editora) de la manera especificada por los autores o por la revista. La licencia completa se puede consultar en:Licencia Creative Commons Atribución-NoComercial-Compartir por igual 4.0 Internacional.
} 